$\boldsymbol{A L F A}$, Revista de Investigación en Ciencias Agronómicas y Veterinarias enero-abril-2017

Volumen 1, Número 1,

pp.38-47

(c) (i) (S) (2)

BY NC SA

\title{
Balance Hídrico en Tres Híbridos Tenera de Palma Aceitera (Elaeis Guineensis Jacq.) Con Riego y Sin Riego
}

\section{Water Balance in Three Hybrid Tenera De Palma Aceitera (Elaeis Guineensis Jacq.) With Irrigation and Without Irrigation}

\section{Katty Ortiz \\ Kortiz1989@hotmail.com \\ Marcelo Calvache}

Universidad Tecnológica Equinoccial, Santo Domingo, Ecuador

Artículo recibido en junio de 2016, arbitrado en julio de 2016 y publicado en enero de 2017

\begin{abstract}
RESUMEN
La palma Aceitera que crece bajo las condiciones de La Concordia sufre un acentuado estrés hídrico, pues soporta relativamente seis meses sin lluvia, entre julio a diciembre. La presente investigación tuvo como propósito conocer la dinámica del agua en el sistema SueloPlanta-Atmósfera para un manejo más eficiente del riego. Hallándose en la evaluación al 90\% de eficiencia diferencias significativas en la variable evapotranspiración para los factores riego e híbridos, siendo el promedio de $2.44 \mathrm{~mm}$ día-1. En el rendimiento no habiendo diferencias estadísticas significativas para los factores en estudio, el híbrido de mayor rendimiento es INIAP con 2.13 t ha-1 año desarrollado en la estación Santo Domingo del INIAP para la zona, seguido por ASD con 18,57 t ha-1año que demuestra adaptación al área de La Concordia y el híbrido de menor eficiencia CIRAD con 15,29 t ha-1 año.
\end{abstract}

Palabras clave: Drenaje; evapotranspiración; almacenamiento; déficit hídrico; riego
ABSTRACT

The Aceitera palm that grows under the conditions of La Concordia undergoes an accentuated hydric stress, since it supports relatively six months without rain, between July to December. The purpose of this research was to know the dynamics of water in the Soil-PlantAtmosphere system for a more efficient irrigation management. Being in the evaluation at $90 \%$ efficiency significant differences in the variable evapotranspiration for the irrigation and hybrid factors, being the average of $2.44 \mathrm{~mm}$ day-1. In the yield, there being no significant statistical differences for the factors under study, the highest yield hybrid is INIAP with $2.13 \mathrm{t}$ ha- 1 year developed in the Santo Domingo station of the INIAP for the area, followed by ASD with $18.57 \mathrm{t}$ ha 1 year that shows adaptation to the La Concordia area and the CIRAD lower efficiency hybrid with 15.29 t ha-1 year.

Key words: Drainage; evapotranspiration; storage; water deficit; irrigation 


\section{INTRODUCCIÓN}

El crecimiento de la población mundial y el acelerado desarrollo económico son factores que mueven la creciente demanda global de aceites y grasas vegetales, el suministro ha mejorado continuamente durante 20 años. Casi todo el crecimiento de la producción ha sido provisto por los aceites vegetales, mientras que la producción de aceites y grasas vegetales se ha estancado durante el mismo período (Fairhurst, 2012).

Durante años la actividad palmicultora en Ecuador se ha venido desarrollando de forma acelerada, llegando a ser uno de los rubros más importantes desde el punto de vista económico-social y como potencial biocombustible, por lo que merece una atención especial dentro del sector agrícola (Herrera, 2011).

La palma de aceite se adapta a períodos de sequía por el cierre de los estomas al medio día, apertura retrasada de hojas y una producción reducida de racimos.El patrón ideal de lluvias es de 2000 a $3500 \mathrm{~mm}$ año, distribuidos uniformemente, con un mínimo de $100 \mathrm{~mm}$ por mes (Fairhurst, 2012).

Conocer la influencia del riego sobre la producción y rentabilidad de palma es; sin lugar a dudas, de gran importancia en zonas con humedad insuficiente (Herrera, 2011). Calculando el Balance Hídrico al determinar la cantidad de agua que entra, que sale y la cantidad que permanece en un determinado volumen de suelo durante el período de crecimiento de un cultivo se establece la eficiencia. Se plantearon los siguientes objetivos:

El objetivo general de este estudio consistió en realizar el cálculo del balance hídrico en tres híbridos Tenera de Palma Aceitera (Elaeis guineensis Jacq.) utilizando la reflectometría en el dominio de la frecuencia (DIVINER 2000) durante la época seca para la zona de La Concordia.

\section{Objetivos específicos}

Estimar el consumo de agua en cada uno de los tres híbridos de Palma Aceitera (Elaeis guineensis Jacq) utilizando el método de Balance de Masas.

Estimar el coeficiente de cultivo (Kc) de los tres híbridos Tenera de Palma Aceitera (Elaeis guineensis Jacq) para la zona de La Concordia.

Relacionar los datos de humedad de suelo y evapotranspiración obtenidos con el DIVINER 2000, los tensiómetros y el lisímetro MC; y, su impacto sobre el rendimiento en los tres híbridos Tenera de Palma Aceitera (Elaeis guineensis Jacq).

\section{MATERIALES Y MÉTODOS}

La investigación se desarrolló en el Centro de Investigaciones de Palma Aceitera (CIPAL) perteneciente a la Asociación Nacional de Cultivadores de Palma Aceitera (ANCUPA), afincado en el Km. $371 / 2$ de la vía Santo Domingo-Quinindé, Cantón La Concordia, Provincia Santo Domingo de los Tsáchilas, entre los meses julio del 2011 y agosto del año 2012.

El material experimental fueron los Híbridos Tenera INIAP, IRHO y ASD de siete años.

El Área Experimental conformada por un total de 18 unidades experimentales la parcela neta consta de 8 híbridos de siete años de edad. Se utilizó un Diseño de Parcela Dividida, el Riego se ubica en la Parcela Grande, mientras que, los materiales se ubican en la Subparcela, con un total de seis interacciones, que se distribuyeron en tres repeticiones.

El drenaje de agua se estableció haciendo uso de la ecuación de Darcy que determina la intensidad de flujo de agua (q) específica de cada humedad volumétrica $(\theta)$.

El almacenamiento de agua se determinó utilizando el DIVINER 2000 que permite conocer con exactitud la cantidad cada $10 \mathrm{~cm}$ de profundidad en el perfil del suelo. 


\section{La evapotranspiración de cultivo se} obtuvo a partir de la ecuación del balance hídrico utilizando las siguientes expresiones:

$$
\mathrm{ETc}=\mathrm{P}+\mathrm{I} \pm \mathrm{D}+\Delta \mathrm{A}
$$

Dónde: $\mathrm{P}=$ precipitación $(\mathrm{mm}) ; \mathrm{D}=$ drenaje (-) o ascensión capilar (+); I = Irrigación (mm) y $\Delta \mathrm{A}=$ variación de almacenamiento de agua en el suelo durante un determinado intervalo de tiempo (mm) y ETc evapotranspiración del cultivo.

Coeficiente de cultivo (Kc) dado por el cociente resultante de relacionar la Evapotranspiración del cultivo ETc y la evapotranspiración de referencia (ETo). Utilizando la siguiente expresión:
ETc
$\mathrm{Kc}=$

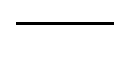
$\mathrm{ETo}=\mathrm{PE}+\mathrm{EMC}$
ETo

Dónde: $\mathrm{PE}=$ Precipitación efectiva; $\mathrm{EMC}=$ Evaporación lisímetro

\section{RESULTADOS Y DISICUSIÓN}

\section{Almacenamiento}

En el Análisis de la Variancia, no se observó diferencias significativas estadísticamente para los factores en estudio. El promedio general del experimento fue de $271.01 \mathrm{~mm}$; el coeficiente de variación (a) para el factor riego fue de $5,20 \%$ y el coeficiente de variación (b) fue de 11,64\%.
En término energéticos, la extracción de agua de las capas profundas es más costoso que la de capas superficiales, hecho que debe tenerse en cuenta en el manejo del riego. Siempre que sea posible, será más favorable para los cultivos mantener alto el nivel de humedad del horizonte superficial, con independencia de que en profundidad exista humedad suficiente para actuar como reserva en caso del agotamiento imprevisto de los niveles superficiales Badillo (2009)

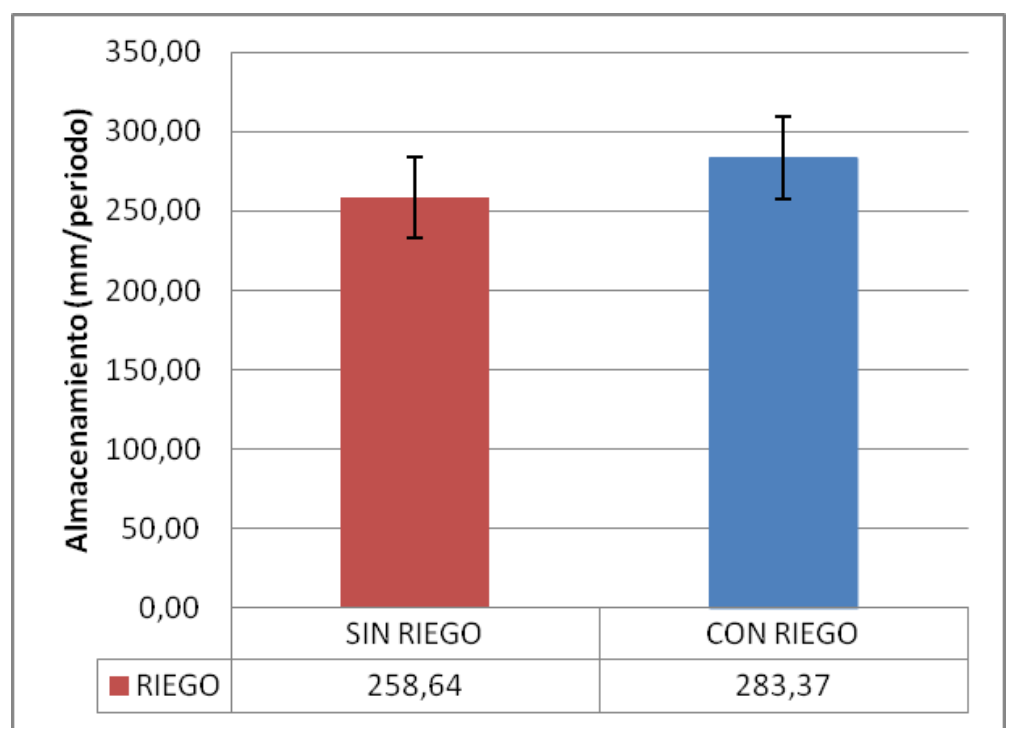

Gráfico 1. Almacenamiento promedio a $80 \mathrm{~cm}$ para el factor. 
Para el factor Riego, gráfico 1, se observa que la mayor respuesta se obtuvo en $\mathrm{r} 1$ (con riego) con un almacenamiento promedio de $283.37 \mathrm{~mm}$, en tanto que, la menor respuesta se obtuvo en r0 (sin riego) con un almacenamiento promedio de $258.64 \mathrm{~mm}$. La capacidad de almacenamiento del suelo es relativa no sólo al tiempo, sino también a la composición textural y a la secuencia de capas del perfil; así como, a la distribución inicial del contenido de aguas Velásquez (2010).

Para el factor Híbridos, Gráfico 2 se observó que el híbrido INIAP presentó la mayor respuesta con un almacenamiento promedio de 283,33 $\mathrm{mm}$; en tanto que, el híbrido ASD presentó la menor respuesta con almacenamiento promedio de $259,10 \mathrm{~mm}$.

Según Chimbo (2011), sobre los resultados de consumo de agua por parte del híbrido INIAP es el de mejor adaptación tiene a la zona donde se realizó el estudio ya que este fue desarrollado por el Instituto Nacional de Investigaciones Agropecuarias, en la Estación Santo Domingo.

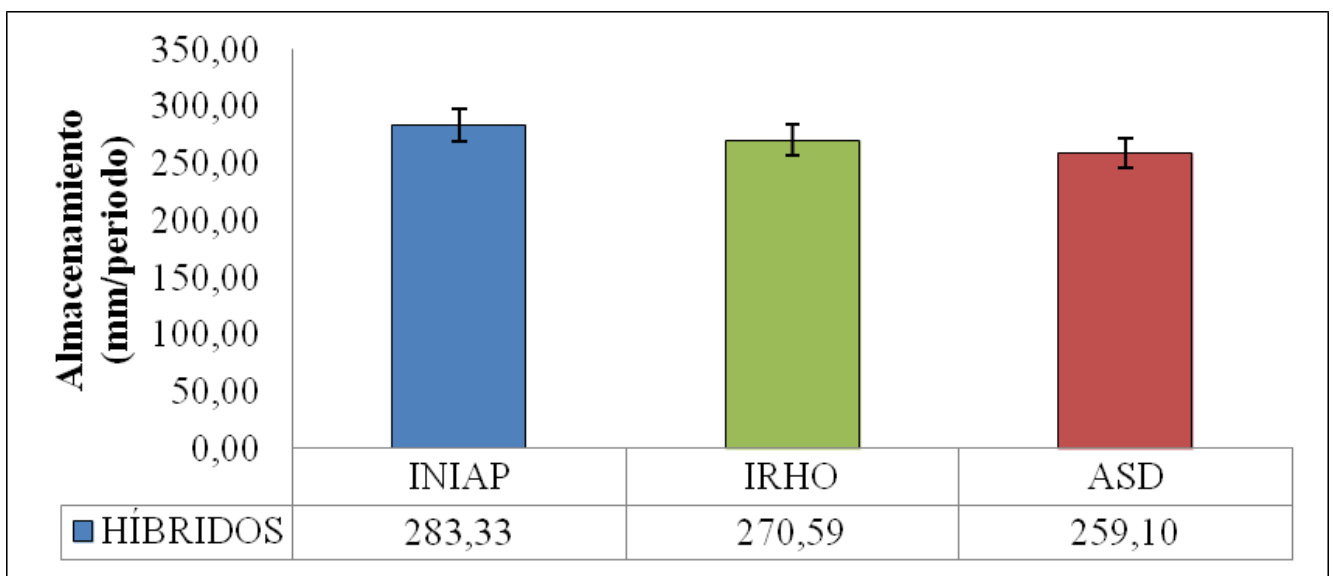

Gráfico 2. Almacenamiento promedio a $80 \mathrm{~cm}$ para el factor Híbrido.

El híbrido ASD se caracteriza por su alto rendimiento que deriva de la mayor producción en número y peso de sus racimos; de ahí, se explica que sea el híbrido que menor almacenamiento presenta con y sin riego. Pero también se ha evidenciado el reducido almacenamiento del híbrido CIRAD con y sin riego, hecho que no es justificable con su producción ya que es el híbrido que menor rendimiento presenta durante el año de evaluación.

\section{Drenaje}

Los resultados del drenaje durante la época de evaluación fueron aproximadamente cero para todas las interacciones en estudio. 
Cuadro 1. Promedios del drenaje a 80 cm en la evaluación del Balance Hídrico.

\begin{tabular}{cc}
\hline FACTORES & $\begin{array}{c}\text { PROMEDIO DE DRENAJE } \\
\text { (mm/día) }\end{array}$ \\
\hline Riego & $1.22 \pm 1.91$ \\
\hline r1 Con Riego & $0.25 \pm 0.51$ \\
r0 Sin Riego & \\
Híbridos & $0.17 \pm 0.22$ \\
h1 INIAP & $2.01 \pm 2.03$ \\
h2 CIRAD & $0.02 \pm 0.03$ \\
h3 ASD & \\
Interacciones & $0.12 \pm 0.20$ \\
r1 h1 (INIAP Con Riego) & $0.22 \pm 0.28$ \\
r0h1 (INIAP Sin Riego) & $3.51 \pm 1.65$ \\
r1h2 (CIRAD Con Riego) & $0.51 \pm 0.88$ \\
r0h2 (CIRAD Sin Riego) & $0.02 \pm 0.03$ \\
r1h3 (ASD Con Riego) & $0.02 \pm 0.04$ \\
r0h3 (ASD Sin Riego) &
\end{tabular}

Chimbo (2011) asevera que la cantidad de agua que se pierde por percolación profunda no tiene ninguna importancia en el almacenamiento que presenta el cultivo, ni va a producir déficit hídrico en el cultivo. El valor que se obtiene como resultado del drenaje total durante los 106 días de investigación es considerablemente menor a los valores de almacenamiento y evapotranspiración.

\section{Evapotranspiración del cultivo}

En el cuadro 2, evapotranspiración del cultivo se consideró una eficiencia del 90\% encontrándose significación estadística para el factor riego y para el factor Híbridos; en tanto que, la interacción $\mathrm{R}$ x $\mathrm{H}$ y repeticiones no existe diferencias estadísticas. El promedio general del experimento fue de $2.44 \mathrm{~mm}_{\text {día }}{ }^{-1}$; el coeficiente de variación (a) fue de $7.28 \%$ y el coeficiente de variación (b) fue de $12.12 \%$. 
Cuadro 2. ADEVA para la evapotranspiración a $80 \mathrm{~cm}$ de profundidad en la evaluación del Balance.

\begin{tabular}{|c|c|c|}
\hline \multirow{2}{*}{$\begin{array}{l}\text { FACTORES } \\
\text { DE VARIACIÓN }\end{array}$} & \multicolumn{2}{|c|}{$\begin{array}{l}\text { CUADRADOS MEDIOS } \\
\text { EVAPOTRANSPIRACIÓ }\end{array}$} \\
\hline & GL & $\begin{array}{l}\text { N } \\
\text { (mm día-1) }\end{array}$ \\
\hline TOTAL & 17 & \\
\hline REPETICIONES & 2 & $0.10 \mathrm{~ns}$ \\
\hline RIEGO & 1 & $2.51^{*}$ \\
\hline ERROR (A) & 2 & 0.03 \\
\hline HÍBRIDOS & 2 & $0.30 *$ \\
\hline $\mathrm{R} \times \mathrm{H}$ & 2 & $0.05 \mathrm{~ns}$ \\
\hline ERROR (B) & 8 & 0.09 \\
\hline Promedio general & & 2.44 mm/día \\
\hline $\mathrm{CV}(\mathrm{a}) \%$ & & $7.28 \%$ \\
\hline $\mathrm{CV}(\mathrm{b}) \%$ & & $12.12 \%$ \\
\hline
\end{tabular}

En el gráfico 3 se observa que la mayor respuesta se obtiene en $\mathrm{r} 1$ (con riego) con un promedio de $2.81 \mathrm{~mm}_{\text {día }}{ }^{-1}$ lo que se debe al riego aplicado; en tanto que, la menor respuesta se obtiene en $\mathrm{r} 0$ (sin riego) con un promedio $2.07 \mathrm{~mm} \mathrm{día}^{-1}$ ).
El estrés sufrido por el cultivo se traduce en la disminución de la tasa de evaporación y, consecuentemente con las funciones fisiológicas que se relacionan con ella: la respiración, la fotosíntesis y la asimilación. Santos (2010).

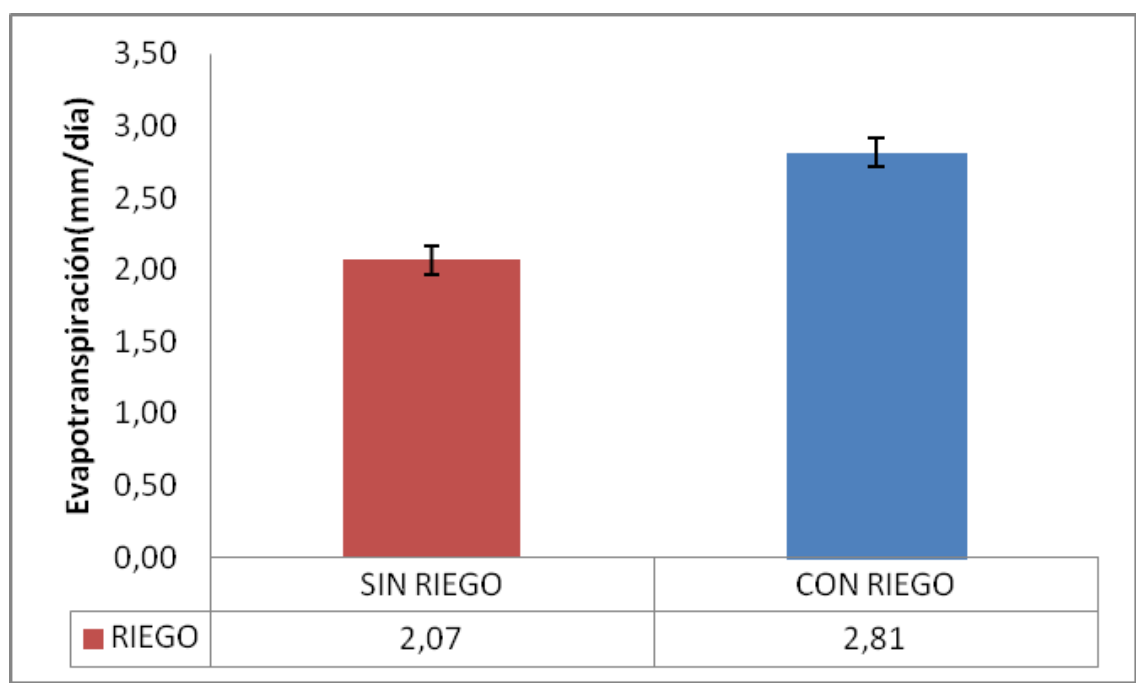

Gráfico 3. Evapotranspiración promedio a $80 \mathrm{~cm}$ de profundidad para el factor Riego. 
Para el Factor Híbridos, gráfico 4, se observó que, el híbrido ASD (h3) presenta la mayor respuesta con una evapotranspiración del cultivo promedio de $2.68 \mathrm{~mm}_{\text {día }}{ }^{-1}$, el $\begin{array}{lllll}\text { híbrido } & \mathrm{h} 1 & \text { (INIAP) } & \text { con } & \text { una } \\ & \end{array}$ evapotranspiración promedio de $7.13 \mathrm{~mm}$ día 1; mientras que, el híbrido CIRAD (h2) presentó la menor respuesta con una evapotranspiración promedio de $2.23 \mathrm{~mm}$ día 1.

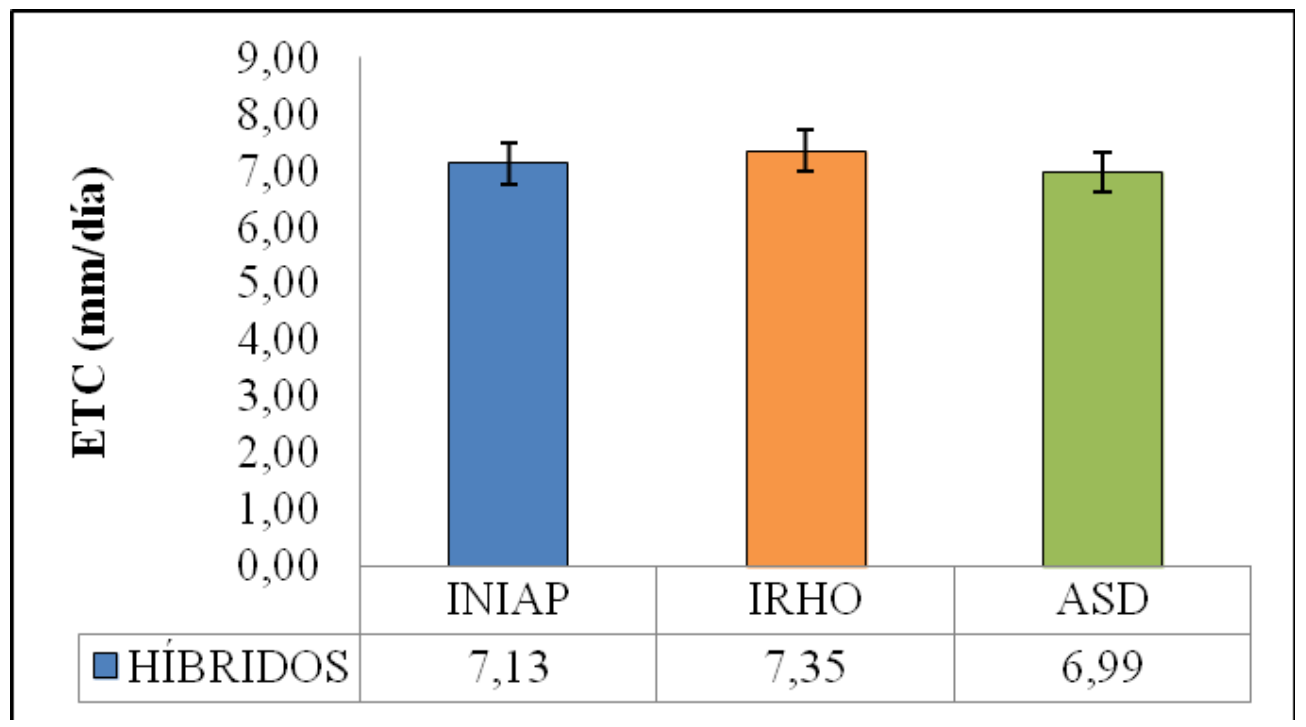

Gráfico 4. Evapotranspiración promedio a $80 \mathrm{~cm}$ de profundidad para el factor Híbridos.

Según Herrera (2012) el agua disponible en el suelo es un factor que influye en la cantidad de agua que es transpirada por la planta, ésta absorbe el agua de acuerdo a su potencial total, el agua se mueve de potenciales mayores hacia potenciales menores y una vez que el agua es absorbida por la planta, se traslada por el xilema para terminar saliendo por los estomas.

\section{Rendimiento}

Para el Factor Híbridos, cuadro 3, gráfico 5, se observa que, al híbrido INIAP (h1) presenta la mayor respuesta con una producción total promedio de 20.13 t/ha/año; mientras que, el híbrido CIRAD (h2) presenta la menor respuesta con una producción total promedio de 15.29 t/ha/año y el ASD con 18.57 t/ha/año.
Vega et al. (2010), Afirma que el riego que se aplica al cultivo no tiene un efecto inmediato sino que su respuesta en la producción es evidente en un lapso de 24 meses; por lo tanto el comportamiento del rendimiento refleja que en el año 2009 no se logró regar el ensayo de acuerdo a las necesidades hídricas del cultivo de palma aceitera (Chimbo, 2011). 
Cuadro 3. Promedios para rendimiento en la evaluación del Balance Hídrico.

\begin{tabular}{ll}
\hline FACTORES & $\begin{array}{l}\text { PROMEDIOS } \\
\text { RENDIMIENTO } \\
\text { (t/ha/año) }\end{array}$ \\
\hline Riego & $18.80 \pm 5.30$ \\
r1 Con Riego & $17.19 \pm 4.25$ \\
r0 Sin Riego & \\
Híbridos & $20.13 \pm 3.53$ \\
h1 INIAP & $18.57 \pm 6.13$ \\
h3 ASD & $15.29 \pm 3.38$ \\
h2 CIRAD & \\
Interacciones & $20.59 \pm 2.38$ \\
r1 h1 (INIAP Con Riego) \\
r0h1 (INIAP Sin Riego) \\
r1h2 (CIRAD Con Riego) \\
r0h2 (CIRAD Sin Riego) & $14.67 \pm 4.87 \pm 1.50$ \\
r1h3 (ASD Con Riego) & $20.10 \pm 7.70$ \\
r0h3 (ASD Sin Riego) & $17.05 \pm 5.28$ \\
\hline
\end{tabular}

Durante este período de evaluación por primera vez desde que se inició el ensayo, el híbrido INIAP presentó el mejor rendimiento, en contraste con los años anteriores donde el mejor híbrido fue ASD.

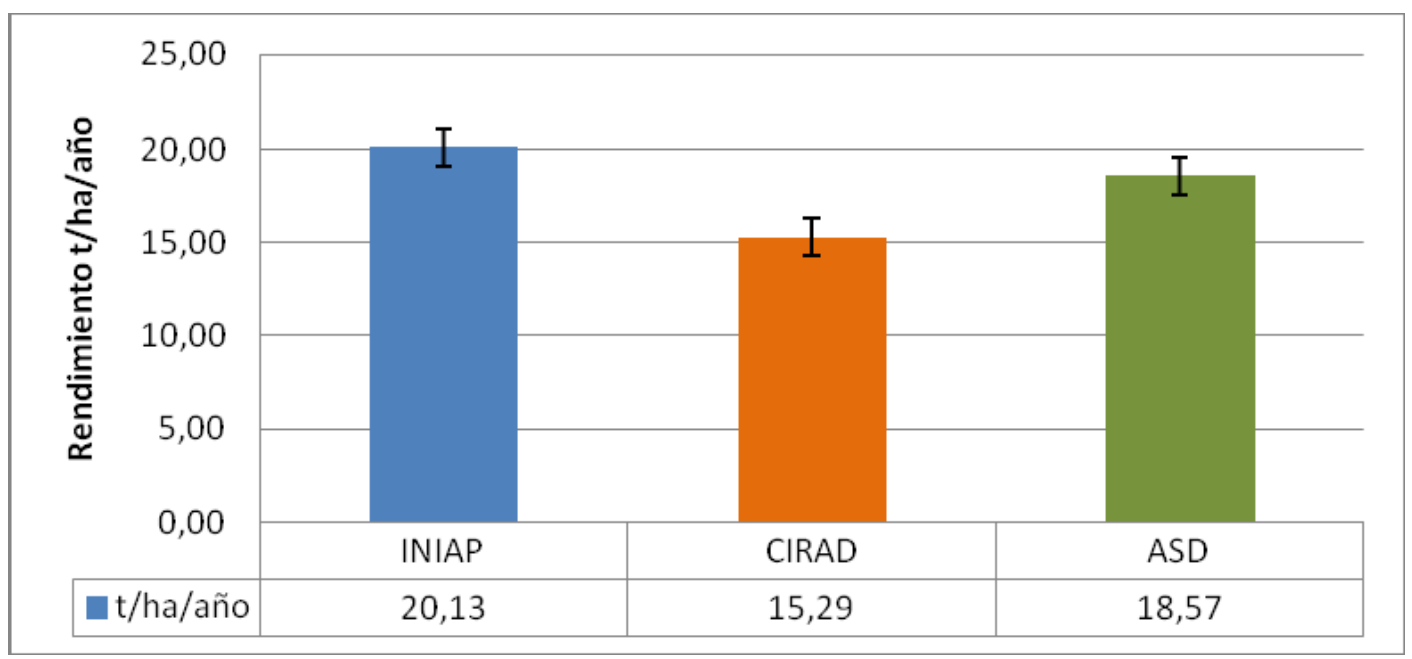

Gráfico 5. Rendimiento promedio para factor Híbrido en la evaluación del Balance. 
Al respecto como lo mencionan Vega et al. (2010), en el año 2009 la poca adaptabilidad del híbrido CIRAD hace que este tenga bajos rendimientos y en contraste los altos rendimientos de ASD, son producidos debido a que este híbrido es un material de la zona, por lo tanto esto ha hecho que se acostumbre y adapte a las condiciones edafoclimáticas del sitio en estudio
La precipitación favorable se cifra entre 1800 y 2200 mm anuales; aunque, se debe tener presente que no basta estudiar la cantidad anual de precipitación, sino su distribución mensual e incluso por décadas, y es aquí donde nuestro sitio experimental tiene ingerencia sobre la producción ya que tenemos aproximadamente 6 a 8 meses de precipitaciones mensuales de menos de 150 mm. (Ver gráfico 6)

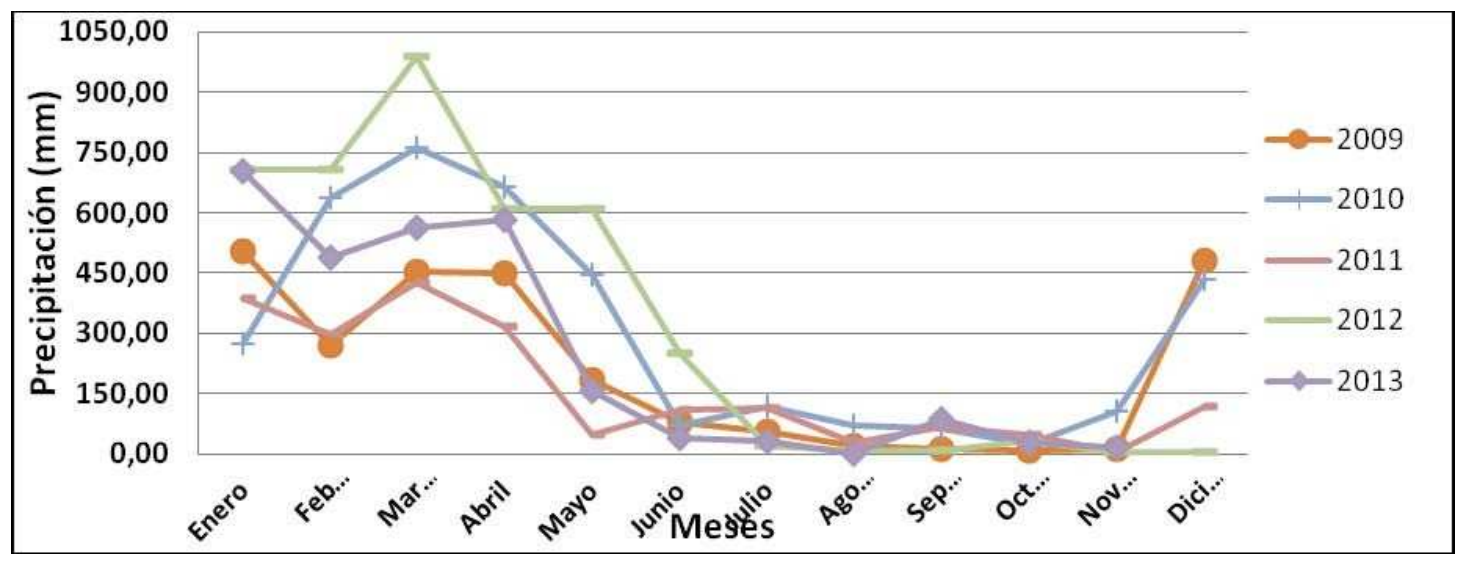

Gráfico 6. Precipitación histórica registrada en el Centro de Investigación de Palma Aceitera.

En el año de investigación, Cuadro 3, los mejores rendimientos se presentaron con la interacción r1h1 (INIAP); mientras que, en años anteriores el mejor rendimiento se presentó en la interacción r1h3 (ASD con Riego) (Lema (2007), Reinoso (2009), Herrera (2012) y Reyes (2012).

\section{Coeficiente del cultivo}

El coeficiente del cultivo describe las variaciones de la cantidad de agua que las plantas toman del suelo, durante sus diferentes estados de crecimiento, desde la siembra hasta la cosecha (Calvache, 2012).

El coeficiente del cultivo obtenido es de 0.95, el cual es representativo y fiable teniendo en cuenta las condiciones climáticas de la zona de La Concordia que se caracteriza por su alta nubosidad. También se reporta el valor del kc obtenido por el método de ETocropwat (Chimbo y Calvache, 2011) que es de 0.73 .

\section{CONCLUSIONES}

- Se logró estimar el consumo de agua según el balance de masa en el híbrido CIRAD (h2) fue de (2.23 mm día-1) y por tanto es el híbrido que menor agua consume, concordando así con su poco crecimiento y baja producción. En tanto que la evapotranspiración que presentó del híbrido INIAP (h1) fue de $(2.41 \mathrm{~mm}$ día-1 ${ }^{-1}$, siendo el híbrido de mayor consumo ASD (h3) con (2.68 mm día-1). El consumo de agua del cultivo de palma es alto, la evapotranspiración del cultivo fue 
inferior en las parcelas sin riego (2.07 $\mathrm{mm}$ día $^{-1}$ ) demostrándose que en estas parcelas se presentó un marcado déficit hídrico durante la época seca; en tanto que, en las parcelas con riego la evapotranspiración promedio fue de 2.81 mm día ${ }^{-1}$.

- Se obtuvo un coeficiente de cultivo promedio para los tres híbridos fue de 0.95 según el resultado obtenido durante el período de evaluación. El kc del híbrido INIAP (h1) fue de 0.97, en tanto que el híbrido CIRAD (h2) presentó un kc de 0.85 y el híbrido ASD (h3) 1.04 .

- El drenaje de las parcelas en estudios fue en promedio de $0.02 \mathrm{~mm}$ día-1 evidenciándose que el agua del perfil del suelo se pierde por evapotranspiración y el consumo mismo que hace la palma aceitera para su mantenimiento, crecimiento y producción. El híbrido INIAP (h1) presentó el mayor almacenamiento (283.33 $\mathrm{mm}$ ) durante el $7 m o$ año de evaluación, en el híbrido CIRAD (h2) fue de (270.59 mm); mientras que el híbrido con menor almacenamiento es ASD (259.10 $\mathrm{mm})$ concluyendo que, es un híbrido de alto uso de agua para sostener su gran crecimiento y alta producción.

- Al relacionar los datos de humedad del suelo y evapotranspiración se observó que es poca la adaptabilidad del híbrido CIRAD lo que se pudo corroborar al observar sus bajos rendimientos durante los años de investigación; lo que contrasta con los altos rendimientos de los híbridos ASD e INIAP que se presentan ya que son materiales de uso común en la zona; por tanto, esto ha hecho que se vaya adaptando a las condiciones edafoclimáticas del sitio en estudio.

\section{REFERENCIAS}

Badillo, M. (2009). Manual de buenas prácticas de riego. Madrid, España. Artes Gráficas Palermo, S.L. 32 p.

Calvache-Ulloa M. (2012). Riego Andino Tecnificado. Primera edición. Quito Ecuador. Editorial Universitaria. 296 p.

Chimbo, G. y Calvache (2011). Balance hídrico de tres híbridos de palma aceitera (ElaeisguineensisJacq.) de seis años de edad. La Concordia, Ecuador. Rumipamba $12 \mathrm{p}$.

Herrera, M., y Calvache, M. (2011). Balance hídrico en tres híbridos tenera de palma aceitera (Elaeisguineensisjacq.) con riego y sin riego. La Concordia, Esmeraldas. Rumipamba CD $10 \mathrm{p}$.

Reinoso, L Y Calvache, M. (2009). Influencia del riego en el comportamiento de tres híbridos de palma aceitera (Elaeisguineensisjacq) de diferentes orígenes (4to años de ejecución) La Concordia, Esmeraldas. Rumipamba CD. $12 \mathrm{p}$

Reyes-Onces, S. y Calvache, M. (2012). Influencia del riego en el comportamiento de tres híbridos de palma aceitera (ElaeisguineensisJacq.) de diferentes orígenes $\left(7^{\circ}\right.$ año de ejecución). La Concordia, Santo Domingo de los Tsáchilas. Rumipamba $8 \mathrm{p}$

Santos, L.; De Juan J.; Picornell M.; Tarjuelo J. (2010). El riego y sus tecnologías. España p 99. Disponible en:

http://www.fagro.edu.uy/ hidrologia/ri ego/El_Riego_y_sus_Tecnologias.pdf (Citado el 17 de enero del 2013)

Velásquez, J., Gómez A. (2010). Palma Africana en Tabasco. México D.F. p16. Disponible en: http://www.archivos.ujat.mx/2011/d ifusion/libros/23.pdf (Citado el 17 de Enero del 2013) 